\title{
Whole-genome sequencing opens a new era for molecular breeding of grass carp (Ctenopharyngodon idellus)
}

\author{
HU Wei* \& CHEN Ji \\ State Key Laboratory of Freshwater Ecology and Biotechnology, Institute of Hydrobiology, Chinese Academy of Sciences, Wuhan 430072, \\ China
}

Received May 5, 2015; accepted May 6, 2015; published online May 6, 2015

Citation: Hu W, Chen J. Whole-genome sequencing opens a new era for molecular breeding of grass carp (Ctenopharyngodon idellus). Sci China Life Sci, 2015, 58: 619-620, doi: 10.1007/s11427-015-4864-x

Recently, the genome sequencing of the grass carp (Ctenopharyngodon idellus) has been jointly completed by researchers from Institute of Hydrobiology (Chinese Academy of Sciences), National Center for Gene Research, and Sun Yat-Sen University. Adopting a whole-genome shotgun sequencing strategy and modified de novo Phusionmeta assembly pipeline, researchers obtained a $0.9 \mathrm{~GB}$ draft genome of a female adult grass carp and a 1.07 GB genome of a wild male. The female adult was selected from an artificial gynogenetic population, with a remarkably higher genome homozygosity in comparison with that of a wild individual. The female genome assembly had scaffolds with an N50 length more than $6.4 \mathrm{Mb}$, reflecting a much higher quality of genome assembly than those in other sequenced aquaculture animals. By comparing RNA-seq data from six tissues of grass carp with homologous gene information from zebrafish, 27,263 protein-coding genes were annotated in the female grass carp genome, of which 17,456 annotated genes could be localized on the linkage groups. Furthermore, the genetic basis for the herbivorous habit of grass carp was discovered through transcriptome analysis. A novel clue associated with sex chromosome differentiation and sex determination in grass carp was found. These findings were published in Nature Genetics [1].

Proteins, lipids, starch and cellulose in food cannot be absorbed by fish unless they are resolved by the digestive enzymes secreted from intestine of fish or intestinal micro-

*Corresponding author (email: huwei@ihb.ac.cn) organisms. Grass carp is so named because of its typically herbivorous habit. The aquatic vascular plants that grass carp eat are cellulose-rich thus hard to be digested. Besides, the content of nutrients easy to be utilizated by fish in the aquatic vascular plants is relatively low. The mechanism by which grass carp obtain nutrients from water grass to support their fast growth has been a key objective of researchers. Wang et al. [1] found that there was no gene encoding for cellulose digestive enzymes in grass carp genome, suggesting that cellulose digestive enzymes in the intestine of grass carp were fully secreted by intestinal microorganisms. This finding clarified the debate over whether herbivorous fish could produce cellulose digestive enzymes themselves. By comparing the transcriptome data of juvenile grass carp before and after the transition from a carnivorous to herbivorous diet, Wang et al. [1] found that the expression patterns of some genes associated with circadian rhythm, appetite control, cell proliferation and differentiation, digestion and metabolism were reset, and several pathways were activated. These results gave a genetic explanation that grass carp could obtain enough nutrients from plants through continuous food intake to meet the demand for their fast growth. Since aquaculture of herbivorous fish is an efficient way of obtaining animal protein, the finding of genetic basis for herbivorous habit of grass carp provided a novel insight into the culture of herbivorous fish species that could utilize plant cellulose efficiently and promoted the development of artificial diets for domesticated carnivorous fish.

Sex is praised as a queen in evolutionary biology. Fish is 
on an evolutionary stage linking invertebrates and higher vertebrates. All types of sex determination in vertebrates have been found in fish, which make fish ideal models to study mechanisms of animal sex determination and differentiation. Moreover, many types of fish show sexual dimorphism in growth rate or body size. For this reason, it is important for fish culture to produce all-females or all-males by using sex-controlling technologies [2]. Up to now, five genes for male determination have been verified in Oryzias latipes (Adrianichthyidae), Takifugu rubripes (Tetraodontidae), Odontesthes hatcheri (Atherinopsidae), Oreochromis niloticus L. (Cichlidae) and several salmonids species (Salmonidae), respectively. However, in species from Cyprinidae, which are the main freshwater aquaculture objects, the study progress of sex determination and differentiation is still slow.

Genes involved in sex determination and differentiation of vertebrates seem to have the tendency to move onto heteromorphic sex chromosomes, which may be a common feature of these genes [3]. By comparing female with male genome of grass carp, Wang et al. [1] identified $2.38 \mathrm{Mb}$ male specific segments including 206 contigs, many of which were located on LG 24. As a member of the Cyprinidae family, grass carp has a chromosome number of $2 n=48$ while zebrafish has a number of $2 n=50$. The gene collinearity and FISH analysis demonstrated that LG 24 of grass carp corresponded to chromosome 10 and 22 of zebrafish, suggesting that a chromosomal fusion occurred during genome evolution of grass carp. LG 24 of grass carp had the longest physical size but the smallest genetic distance, reflecting a remarkably lower rate of crossing over in meiosis than other linkage groups. Usually, partial unpairing and low recombination rate are observed for sex chromosomes, due to sex specific genes or DNA fragments on them. Given this view, the formation of LG 24 of grass carp was surmised to be associated with sex differentiation, and LG 24 has the potential to be heteromorphic sex chromosomes, with sex- determination genes on them. Up to now, most of confirmed male determinant genes are copies of $d m r t 1$ gene [4], while the male specific DNA fragment isolated by Wang et al. seemed not to have homologs in any other vertebrates, suggesting its unique origin in grass carp. This finding provides a novel clue to study the mechanisms of sex determination and differentiation in Cyprinidae species.

Fish are the last wild food available for human, and aquaculture is considered to be the only way to meet the growing demand for aquatic animal protein [5]. As one of the most important aquaculture species, grass carp accounts for $15.6 \%$ of the global freshwater aquaculture production in 2011. In China, most of freshwater aquaculture species are from Cyprinidae, and species with annual production above 2.5 million tons are all from Cyprinidae. Since grass carp is a representative of Cyprinidae family, the draft genome of grass carp will not only lay the foundations for the study of evolution of fish genome and mechanisms of sex determination and differentiation, but also help explore genes associated with economically important traits and develop new fish varieties with characters such as high yield, disease resistance and high feed conversion rate.

1 Wang YP, Lu Y, Zhang Y, Ning ZM, Li Y, Zhao Q, Lu HY, Huang R, Xia XQ, Feng Q, Liang XF, Liu KY, Zhang L, Lu TT, Huang T, Fan DL, Weng QJ, Zhu CR, Lu YQ, Li WJ, Wen ZR, Zhou CC, Qilin Tian QL, Kang XJ, Shi MJ, Zhang WT, Jang SH, Du FK, He S, Liao LJ, Li YM, Gui B, He HH, Ning Z, Yang C, He LB, Luo LF, Yang R, Luo Q, Liu XC, Li SS, Huang W, Xiao L, Lin HR, Han B, Zhu ZY. The draft genome of the grass carp (Ctenopharyngodon idellus) provides genomic insights into its evolution and vegetarian diet adaptation. Nat Genet, 2015, doi: 10.1038/ng.3280

2 Chen J, Hu W, Zhu ZY. Progress in studies of fish reproductive development regulation. Chinese Sci Bull, 2013, 58:7-16

3 Van Doom GS, Kirkpatrick M. Turnover of sex chromosomes induced by sexual conflict. Nature, 2007, 449: 909-912

4 Matson CK, Zarkower D. Sex and the singular DM domain: insights into sexual regulation, evolution and plasticity. Nat Rev Genet, 2012, 13:163-174

5 Cressey D. Future fish. Nature, 2009, 458: 398-400

Open Access This article is distributed under the terms of the Creative Commons Attribution License which permits any use, distribution, and reproduction in any medium, provided the original author(s) and source are credited. 